\section{Genetic risk of depression and stress-induced negative affect in daily life}

\author{
MARIEKE WICHERS, INEZ MYIN-GERMEYS, NELE JACOBS, \\ FRENK PEETERS, GUNTER KENIS, CATHERINE DEROM, \\ ROBERT VLIETINCK, PHILIPPE DELESPAUL and JIM VAN OS
}

type of environmental stressors that are associated with depression in men and women (Kendler et al, 2001), and evidence for gender-specific genetic factors for both neuroticism and depression (Fanous et al, 2002).

\section{Experience sampling method}

The experience sampling method (ESM) is a structured diary technique to assess people in their daily living environment and has been validated for the purpose of studying the immediate effects of stressors on mood (Csikszentmihalyi \& Larson, 1987; DeVries, 1992; Delespaul, 1995). Participants received a digital wristwatch and a set of ESM self-assessment forms collated in a booklet for each day. The wristwatch was programmed to emit a signal ('beep') at an unpredictable moment in each of ten 90 min time blocks between 07.30 and 22.30 on five consecutive days. After each beep participants were required to stop their activity and to fill out the ESM self-assessment forms previously handed to them, collecting reports of thoughts, current context (activity, persons present and location), appraisals of current situation and mood. All self-assessments were rated on seven-point Likert scales. Trained research assistants with ample experience in momentary assessment technique explained the ESM procedure to the participants during an initial briefing session and a practice form was completed to confirm that the latter were able to understand the Likert scale. Participants were given a telephone number to call in case they had questions or problems during the ESM sampling period. They were instructed to complete their reports immediately after the beep, thus minimising memory distortion, and to record the time at which they completed the form. In order to know whether the participants had completed the form within $15 \mathrm{~min}$ of the beep, the time at which they indicated they completed the report was compared with the actual time of the beep. All reports not filled in within $15 \mathrm{~min}$ after the beep were excluded from the analysis, since previous work (Delespaul, 1995) has shown that reports completed after this interval are less reliable and consequently less valid. In addition, participants with fewer than 17 valid reports (out of 50) were excluded from the analysis, as previous work has shown that measures of individuals with 
less than $30 \%$ of completed reports are unreliable (Delespaul, 1995).

\section{Measurements}

The Structured Clinical Interview for DSM-IV Axis I disorders (SCID; First et $a l, 1996)$ was administered in order to obtain current and lifetime diagnoses of major depressive disorder. Participants also filled in the Symptom Check List - 90 - Revised (SCL-90-R; Derogatis, 1983) in order to obtain a continuous measure of depressive symptoms. The SCL-90-R depression score was log-transformed in order to improve normality.

Measures of stress and negative affect were collected at each beep within the experience sampling method framework. In order to measure ESM event-related stress (hereafter referred to simply as 'stress'), participants were asked to report the most important event that happened between the current and the previous beep. This event was subsequently rated on a sevenpoint bipolar scale $(-3$ very unpleasant, 0 neutral, 3 very pleasant). Responses were recoded to allow high scores to reflect stress ( -3 very pleasant, 0 neutral, 3 very unpleasant). Negative affect was assessed at each beep with six mood adjectives ('insecure', 'lonely', 'anxious', 'low', 'guilty' and 'suspicious') rated on seven-point Likert scales as described above. The mean of the six items was taken as the measure of negative affect (Cronbach's $\alpha=0.76$ over the participants' mean).

\section{Cross-twin, cross-trait method}

The cross-twin, cross-trait method was used in this study. This means that within a twin pair, variable $x$ in the proband is associated with variable $y$ in the co-twin (Fig. 1). The reason why the second variable is measured in the other twin is because measure $x$ may be confounded by measure $y$ when measured in the same person. This method thus ensures that the association between $x$ and $y$-or in this case 'negative mood bias' and 'depression' - is uncontaminated.

\section{Analyses}

Experience sampling method data have a hierarchical structure. In this study, multiple observations (level 1) were clustered within individuals (level 2), who were part of twin pairs (level 3). Multilevel

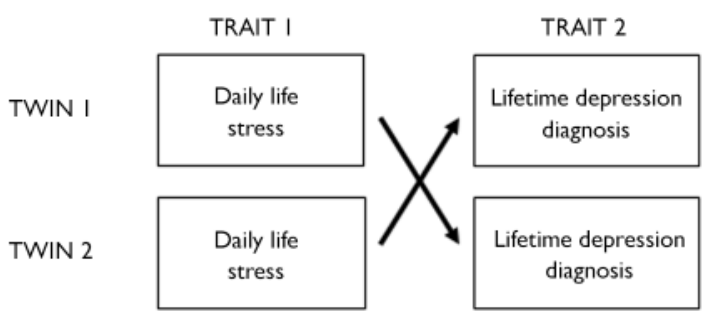

Fig. I Cross-twin, cross-trait design: examination of the interaction term formed by (trait I twin I) $\times$ (trait 2 twin 2) and vice versa.

analysis takes the variability associated with each level of nesting into account (Snijders \& Bosker, 1999).

In order to investigate the association between stress and negative affect in the course of daily life (i.e. stress sensitivity), multilevel linear regression analyses, using the XTMIXED command in Stata version 9.1 for Windows, were applied to the data. A cross-twin, cross-trait design was used in which negative affect in the proband twin was regressed on the interaction 'stress' of the proband and lifetime DSM-IV diagnosis of major depressive disorder in the co-twin (hereafter referred to as co-twin lifetime depression), to test the hypothesis that familial vulnerability for depression would have an impact on stress sensitivity (i.e. would moderate the association between stress and negative affect). The analysis was additionally corrected for the SCL-90-R depression score and past DSM-IV depressive disorder in the proband twin, and a sensitivity analysis was carried out excluding all proband twins with a current depressive state.

Next, the degree of dose-response relationship in the association between stress and negative affect as a function of co-twin lifetime depression was investigated, with the hypothesis that higher appraisals of stress would display greater interaction effects. Thus, it was examined whether higher stress appraisal was associated with a greater impact of co-twin lifetime depression on stress reactivity, the effect of stress on negative affect. For this purpose, using the score 'very pleasant' as the reference category, six dummies were created, since the appraisal scores for the stress of the event ranged from -3 to 3 ('very pleasant' to 'very unpleasant'). From the model with the interactions between the stress dummy variables and co-twin lifetime depression, effect sizes were calculated for stress with and without co-twin lifetime depression, stratified for each separate level of stress by applying and testing the appropriate linear combinations using the Stata
LINCOM command. Main effects and interactions were assessed by Wald test (Clayton \& Hill, 1993).

Finally, in order to examine possible contribution of genes to observed crosstwin, cross-trait associations, the threeway interaction Stress $\times$ co-twin lifetime depression $\times$ zygosity was fitted and evaluated, followed by calculation of stratified effect sizes using the LINCOM command.

\section{RESULTS}

The total sample consisted of 621 White female participants. Thirty-one women were excluded because they had fewer than 17 valid ESM self-reports or missing ESM self-reports. Forty-five women were nontwin sisters and therefore were not included in the analyses. Another 34 participants were excluded because of missing data. This resulted in a data-set of 511 participants who were part of 259 different twin pairs, of which 158 were monozygotic, 100 were dizygotic and 1 was of unknown zygosity. The mean age of the twins was 27 years (s.d. $=7.4$, range $18-46)$. Nearly twothirds $(63 \%)$ had a college or university degree, $35 \%$ had completed secondary education and $2 \%$ had primary education only. The majority were currently employed (63\% employed, $32 \%$ student, $2 \%$ unemployed, $2 \%$ homemaker and $0.4 \%$ sick leave).

\section{Depression vulnerability and negative affect reactivity to daily life stress}

The total number of ESM observations was 14323 and the mean number of observations within individuals was 28 . Eightynine probands $(\mathbf{1 7 . 4 \% )}$ ) had a co-twin with a diagnosis of lifetime depression. The mean stress score was -1.00 (s.d. $=0.62$ ) for participants with co-twin lifetime depression and -1.16 (s.d. $=0.75$ ) for those without. A multilevel analysis showed no significant association between proband 
stress and co-twin lifetime depression $\left(\chi^{2}=1.62, P=0.2\right)$. The mean negative affect score was $0.82($ s.d. $=0.19)$ and $0.82(0.23)$ respectively for the two groups. The association between co-twin lifetime depression and proband mean negative affect was not significant $\left(\chi^{2}=1.66, P=0.2\right)$.

Multilevel analyses showed a significant main effect of stress on negative affect ( $\beta=0.034, P<0.001)$. There was a significant interaction between proband stress and co-twin lifetime depression in the association with negative affect $\left(\chi^{2}=15.9\right.$, $P=0.0001$ ), which remained significant after correction for proband SCL-90-R depression score and proband past depressive disorder $\left(\chi^{2}=16.2, P=0.0001\right)$ and after additionally excluding all probands with current depression according to SCID interview ( $\left.n=482 ; \chi^{2}=9.95, P=0.002\right)$.

\section{Dose-response relationship}

Compared with the baseline of 'very pleasant', events that were rated as 'pleasant' $\left(\chi^{2}=0.01, P=0.92\right)$ or 'slightly pleasant' $\left(\chi^{2}=2.07, P=0.15\right)$ did not interact with co-twin lifetime depression. The interaction effect size increased for events rated as 'neutral' $\left(\chi^{2}=3.49, P=0.06\right)$ and 'slightly unpleasant' $\left(\chi^{2}=10.5, P=0.001\right)$ and was smaller again for events rated as 'unpleasant' and 'very unpleasant' (respectively $\chi^{2}=1.25, P=0.26$, and $\chi^{2}=5.06$, $P=0.02)$. Stratified effect sizes calculated with the LINCOM procedure are displayed in Fig. 2 (the numbers of observations and participants are detailed in Table 1). The two lines represent the effect sizes of stress on negative affect: one line includes and the other does not include the interaction term of stress $\times$ co-twin lifetime depression. Thus, the difference between the two lines represents the size of the interaction effect.

\section{Genetic contribution to the observed cross-twin, cross-trait relationship}

There was a significant three-way interaction between zygosity, co-twin lifetime depression and stress in the association with negative affect $\left(\chi^{2}=6.73, P=0.010\right)$, indicating that the interaction effect between co-twin lifetime depression and stress was significantly stronger in monozygotic twin pairs $(\beta=0.026, P<0.001)$ than in dizygotic pairs $(\beta=0.003, P=0.7)$. Effect sizes stratified by zygosity calculated with the LINCOM procedure are displayed in

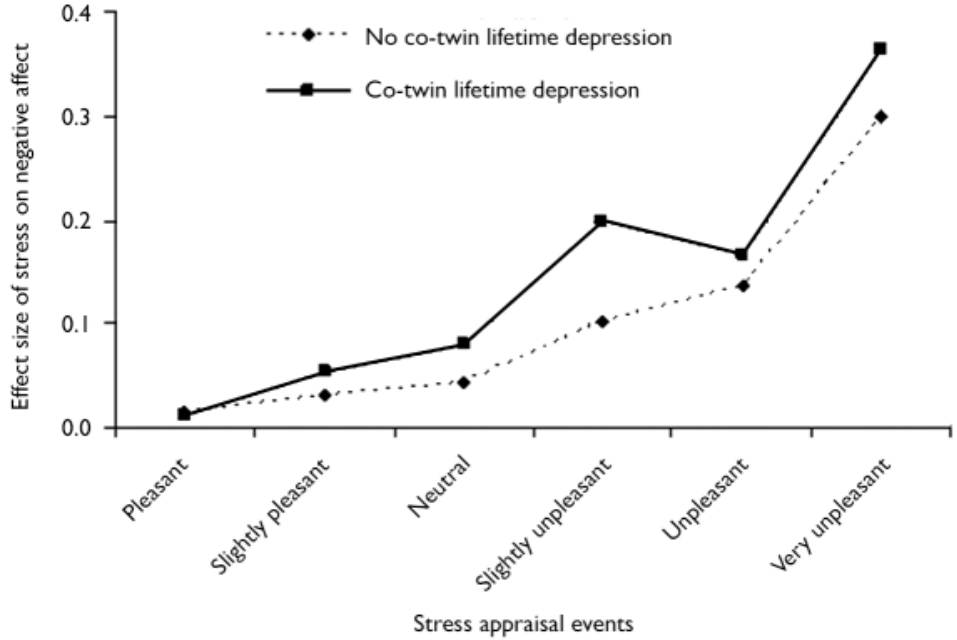

Fig. 2 Effect sizes of stressful events on negative affect: effect sizes of the affective response of stress levels 'pleasant' to 'very unpleasant' relative to the affective response of the reference category 'very pleasant', stratified by co-twin lifetime depression and corrected for continuous depression score.

Table I Number of observations for each level of stress appraisal separate for subjects with and without co-twin lifetime depression, and the number of participants contributing to each number of observations (see Fig. 2)

\begin{tabular}{lrrrrrrrr}
\hline & \multicolumn{7}{c}{ Event stress appraisal } \\
\cline { 2 - 8 } & -3 & -2 & -1 & 0 & 1 & 2 & 3 & Total \\
\hline Co-twin with depression & & & & & & & \\
$\quad$ Observations, $n$ & 510 & 579 & 399 & 720 & 130 & 79 & 96 & 2513 \\
$\quad \begin{array}{l}\text { Participants, } n \\
\text { Co-twin without depression }\end{array}$ & 85 & 81 & 71 & 80 & 56 & 48 & 41 & 88 \\
$\quad$ Observations, $n$ & 2956 & 2517 & 1910 & 2999 & 576 & 412 & 361 & 11731 \\
$\quad$ Participants, $n$ & 359 & 376 & 341 & 372 & 261 & 200 & 190 & 418 \\
\hline
\end{tabular}

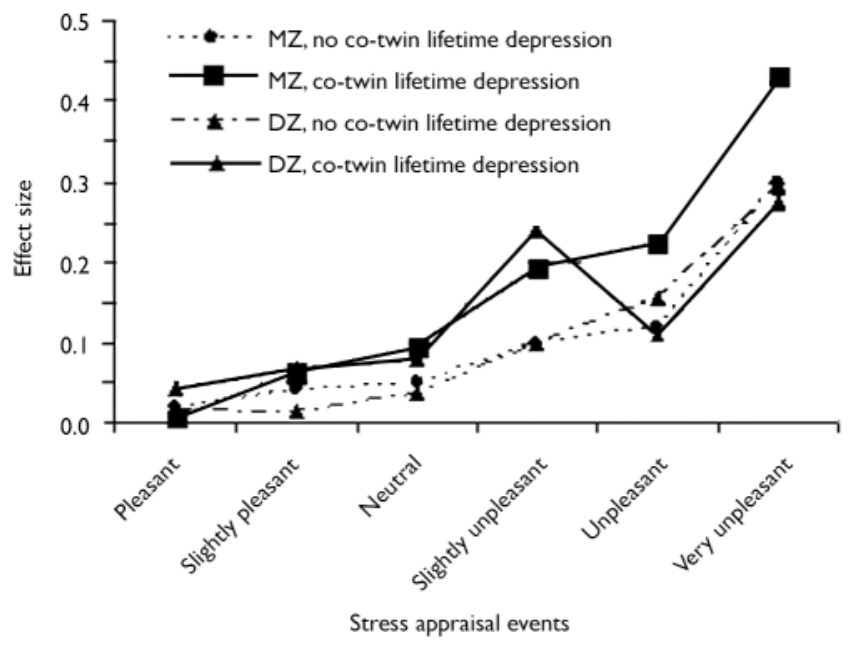

Fig. 3 Associations between stressful events and negative affect, stratified by zygosity and co-twin lifetime depression, corrected for proband continuous depression score and proband depressive disorder in the past (DZ, dizygotic; MZ, monozygotic). 
Table 2 Number of observations and corresponding number of participants for each level of stress appraisal for monozygotic and dizygotic participants with and without a co-twin with lifetime major depression (see Fig. 3)

\begin{tabular}{|c|c|c|c|c|c|c|c|c|}
\hline & \multicolumn{8}{|c|}{ Event stress appraisal } \\
\hline & -3 & -2 & -1 & 0 & I & 2 & 3 & Total \\
\hline \multicolumn{9}{|l|}{ Monozygotic twins } \\
\hline \multicolumn{9}{|c|}{ Co-twin with depression } \\
\hline Observation, $n$ & 332 & 328 & 238 & 365 & 94 & 48 & 65 & 1470 \\
\hline Participants, $n$ & 45 & 45 & 40 & 43 & 35 & 29 & 26 & 48 \\
\hline \multicolumn{9}{|c|}{ Co-twin without depression } \\
\hline Observations, $n$ & 1922 & 1612 & 1168 & 1953 & 346 & 255 & 219 & 7475 \\
\hline Participants, $n$ & 232 & 233 & 216 & 234 & 166 & 126 & 123 & 262 \\
\hline \multicolumn{9}{|l|}{ Dizygotic twins } \\
\hline \multicolumn{9}{|c|}{ Co-twin with depression } \\
\hline Observations, $n$ & 178 & 251 & 161 & 355 & 36 & 31 & 31 & 1043 \\
\hline Participants, $n$ & 40 & 36 & 31 & 37 & 21 & 19 & 15 & 40 \\
\hline \multicolumn{9}{|c|}{ Co-twin without depression } \\
\hline Observations, $n$ & 1027 & 882 & 725 & 1030 & 225 & 153 & $|4|$ & 4183 \\
\hline Participants, $n$ & 126 & 140 & 123 & 135 & 92 & 73 & 66 & 153 \\
\hline
\end{tabular}

Fig. 3 (the numbers of observations and participants are detailed in Table 2).

\section{DISCUSSION}

\section{Negative mood bias endophenotype}

Probands with the greatest level of familial liability for depression displayed greater negative affect responses toward daily life stress. This finding cannot be accounted for by higher current depression score or diagnosis of depression in the proband since the interaction with co-twin depression persisted after controlling for proband continuous depression score and past depressive disorder, and additionally excluding probands with current depression. Thus, increased negative affect reactivity, measured as negative affect level in response to daily life stress, is also present in participants with high familial loading regardless of past or current depression. In addition, a partial dose-response relationship was found: appraisals of greater stressfulness associated with events were associated with increased interaction effects in the negative affect model. For the most stressful events the difference between the groups was smaller than for events that were appraised as less stressful; numbers, however, were smaller in the former. The conservative conclusion is that events that are moderately stressful cause very little negative affect in control participants; in people with a high family loading of depression, however, these events induce significant amounts of negative affect. When stratified by zygosity, however, the results become more conclusive, as in Fig. 3 it can be seen that for monozygotic twins also differences exist for major stressors. In dizygotic twins, however, similar ratings of negative affect reactivity exist for major stressors regardless of the depression status of the co-twin. Thus, for major stressors to show a difference in affective response, a certain degree of genetic vulnerability for depression (on average higher for monozygotic than for dizygotic probands with a depressed co-twin) may be necessary (see below). The findings suggest that in healthy people the underlying trait vulnerability to depression may become evident in the way they react affectively to the small stresses of everyday life. Furthermore, it can be concluded that such a trait, representing a mood bias toward negative affect in the face of stress, is state-independent and thus a likely endophenotype of depression.

This endophenotype may have importance also in predicting vulnerability to other psychiatric disorders apart from depression. In a study examining emotional reactivity to daily stress in psychosis, it was found that patients with psychosis, in comparison with a control group, also showed increased negative affect reactivity to stress in response to daily life stressors. In addition, a separate group of first-degree relatives of patients with psychosis showed intermediate levels of stress sensitivity (Myin-Germeys et al, 2001). These data suggest that the mood bias endophenotype may transcend the borders of traditional diagnostic classification.

\section{Negative mood bias and genetic transmission}

Our data additionally suggest that genetic factors are likely to have a role, and thus provide a link between genetic factors and a phenotype consisting of an interaction between individuals and their environment in the course of daily life. The finding is in accordance with a previous study in the same sample that found, using structural equation modelling, that the association between daily stressors and negative affect was influenced by genetic factors (Jacobs et al, 2006). The analyses suggest that the mood bias trait fulfils the criteria for endophenotype such as heritability and familial association. Whether the trait additionally shows specificity and co-segregation in families, another requisite of the 'endophenotype' definition (Gottesman \& Gould, 2003), should become the subject of further investigation. The link between genetic factors and a person-environment momentary assessment phenotype may be helpful in tracing the link from genotype to clinical depression and may be productively further examined using molecular genetic approaches. The data show that genetic effects can be thought of as influencing person-environment interactions rather than rigidly defined psychopathological phenotypes, and underline the importance of including environmental measurements in genetic approaches towards psychiatric disorders (Kendler, 2005; Moffitt et al, 2005; Rutter, 2005). Although the aim of the investigation was to examine the possible contribution of genetic factors to the mood bias phenotype - and evidence for such a role was found - a role of shared environmental factors is also likely. For example, had we found that negative affective response to stress was increased in people with a cotwin with depression and that this association was of the same magnitude in monozygotic and dizygotic pairs, then the conclusion would have been that only shared environmental factors contribute to the mood bias. The fact that the association was significantly greater within monozygotic pairs than in dizygotic pairs 
provides positive evidence for the involvement of genetic factors, but does not rule out a contribution from the shared environment.

\section{Strengths of the study}

Strengths of the study include the fact that it was the first to use a prospective momentary assessment design in an attempt to capture a mood phenotype at the conceptual level at which it is defined: reactivity in the course of daily life. The cross-twin, cross-trait design allowed for assessment of unconfounded relationships and assessment of the role of genetic factors. Another important strength was the separate assessment of compliance and validation of the ESM procedure as published elsewhere (Jacobs et al, 2005).

\section{Limitations}

Some methodological limitations are apparent. First, it has been suggested that problems may arise in the ESM procedure as it depends on the compliance of participants (Kudielka et al, 2003; Broderick et al, 2004). In particular, fixed time sampling protocols may be problematic and can bias results. However, this report did not use a fixed time sampling frame, and our ESM procedure was validated in a previous report. Thus, the same sample as described in this analysis (Jacobs et al, 2005) was instructed to take, during the ESM procedure, saliva samples at each of the ten unpredictable moments during the five consecutive days. Participants recorded collection times, unaware that compliance with the sampling protocol was being investigated by means of electronic monitoring devices. Results showed that compliance was high (over 90\%) and inclusion of the inaccurately timed samples did not distort the data (Jacobs et al, 2005). Therefore, results from the ESM procedure in this report can be considered valid.

Another issue is that higher levels of negative affect itself may represent the real vulnerability factor, rather than negative affect reactivity to stress. Healthy probands with co-twin siblings who have a lifetime diagnosis of depression may show increased levels of negative affect compared with those whose co-twin has no such diagnosis; higher levels of negative affect give rise to more variability, which in turn enhances the detection of stress sensitivity. However, no difference in negative affect was apparent between participants with and

MARIEKE WICHERS, MA, PhD, INEZ MYIN-GERMEYS, MA, PhD, Department of Psychiatry and Neuropsychology, South Limburg Mental Health Research and Teaching Network, EURON, Maastricht University, Maastricht, The Netherlands; NELE JACOBS, MA, PhD, Department of Psychiatry and Neuropsychology, South Limburg Mental Health Research and Teaching Network, EURON, Maastricht University, Maastricht and Faculty of Psychology, Open University of The Netherlands, Heerlen; FRENK PEETERS, MD, PhD, GUNTER KENIS, MSc, PhD, Department of Psychiatry and Neuropsychology, South Limburg Mental Health Research and Teaching Network, EURON, Maastricht University, Maastricht; CATHERINE DEROM, MSc, PhD, ROBERT VLIETINCK, MD, PhD, Department of Human Genetics, University Hospital Gasthuisberg, Catholic University of Leuven, Leuven, Belgium; PHILIPPE DELESPAUL, MA, PhD, Department of Psychiatry and Neuropsychology, South Limburg Mental Health Research and Teaching Network, EURON, Maastricht University, Maastricht; JIM VAN OS, Department of Psychiatry and Neuropsychology, South Limburg Mental Health Research and Teaching Network, EURON, Maastricht University, Maastricht, The Netherlands, and Division of Psychological Medicine, Institute of Psychiatry, London, UK

Correspondence: Correspondence: M.C.Wichers, Department of Psychiatry and Neuropsychology, South Limburg Mental Health Research and Teaching Network, EURON, Maastricht University, Vijverdalseweg I, Concorde Building, Maastricht, The Netherlands. Tel: +31 433688669 ; fax: +3l 43368 8689; email: m.wichers@sp.unimaas.nl

(First received 12 October 2006, final revision 23 February 2007, accepted 30 April 2007)

without family loading for depression and this explanation is therefore unlikely. Furthermore, this study was longitudinal but essentially assessed multiple crosssectional relationships at each ESM moment, which made it impossible to establish causal relationships. Therefore, it is impossible to determine whether stress measures influence mood, or whether mood influences subjective appraisals of stress. However, either explanation bears clinical relevance, and the interpretation of stress at least in part contributing to measures of mood has face validity. Finally, since only women were included in this study, the results are not necessarily generalisable to the male population.

\section{ACKNOWLEDGEMENTS}

This research was supported by the Dutch Foundation for Scientific Research. The East Flanders Prospective Survey has been in part supported by grants from the Fund of Scientific Research, Flanders, and Twins, the Association for Scientific Research in Multiple Births, Belgium. We thank all the participating twins for their cooperation.

\section{REFERENCES}

Bolger, N. \& Schilling, E. A. (1991) Personality and the problems of everyday life: the role of neuroticism in exposure and reactivity to daily stressors. Journal of Personality, 59, 355-386.

Broderick, J. E., Arnold, D., Kudielka, B. M., et al (2004) Salivary cortisol sampling compliance: comparison of patients and healthy volunteers. Psychoneuroendocrinology, 29, 636-650.
Clayton, D. \& Hill, M. (1993) Wald tests. In Statistical Models in Epidemiology (eds D. Clayton \& M. Hills), pp. 101-102. Oxford Science Publications.

Csikszentmihalyi, M. \& Larson, R. (1987) Validity and reliability of the Experience-Sampling Method. Journal of Nervous and Mental Disease, 175, 526-536.

Delespaul, P. (1995) Assessing Schizophrenia in Daily Life: The Experience Sampling Method. University of Limburg.

Derogatis, L. R. (1983) $S C L-90-R$ : Administration, Scoring and Procedures Manual - II. Clinical Psychometric Research.

Derom, C., Vlietinck, R., Thiery, E., et al (2002) The East Flanders Prospective Twin Survey (EFPTS). Twin Research, 5, 337-34I.

DeVries, M.W. (1992) The Experience of Psychopathology: Investigating Mental Disorders in Their Natural Settings. Cambridge University Press.

Erickson, K., Drevets, W. C., Clark, L., et al (2005)

Mood-congruent bias in affective go/no-go performance of unmedicated patients with major depressive disorder. American Journal of Psychiatry, 162. 2171-2173.

Fanous, A., Gardner, C. O., Prescott, C. A., et al (2002) Neuroticism, major depression and gender: a population-based twin study. Psychological Medicine, 32. 719-728.

First, M. B., Gibbon, M., Spitzer, R. L., et al (1996) User's Guide for the Structured Interview for DSM-IV Axis I Disorders - Research Version. Biometrics Research Institute.

Gottesman, I. I. \& Gould, T. D. (2003) The endophenotype concept in psychiatry: etymology and strategic intentions. American Journal of Psychiatry, 160, 636-645.

Gunthert, K. C., Cohen, L. H. \& Armeli, S. (1999)

The role of neuroticism in daily stress and coping. Journal of Personality and Social Psychology, 77, 1087-1100.

Hasler, G., Drevets, W. C., Manji, H. K., et al (2004)

Discovering endophenotypes for major depression. Neuropsychopharmacology, 29, 1765-1781.

Hettema, J. M., Neale, M. C., Myers, J. M., et al (2006) A population-based twin study of the relationship between neuroticism and internalizing disorders. American Journal of Psychiatry, 163, 857-864. 
Horwood, L. J. \& Fergusson, D. M. (1986)

Neuroticism, depression and life events: a structural equation model. Social Psychiatry, 2I, 63-7I.

Jacobs, N., Nicolson, N. A., Derom, C., et al (2005) Electronic monitoring of salivary cortisol sampling compliance in daily life. Life Sciences, 76, 2431-2443.

Jacobs, N., Rijsdijk, F., Derom, C., et al (2006) Genes making one feel blue in the flow of daily life: a momentary assessment study of gene-stress interaction. Psychosomatic Medicine, 68, 20I-206.

Kendler, K. S. (2005) Psychiatric genetics: a methodologic critique. American Journal of Psychiatry, 162, 3-II.

Kendler, K. S., Thornton, L. M. \& Prescott, C. A (200I) Gender differences in the rates of exposure to stressful life events and sensitivity to their depressogenic effects. American Journal of Psychiatry, 158, 587-593.
Kendler, K. S., Kuhn, J. \& Prescott, C. A. (2004) The interrelationship of neuroticism, sex, and stressful life events in the prediction of episodes of major depression. American Journal of Psychiatry, 161, 631-636.

Kudielka, B. M., Broderick, J. E. \& Kirschbaum, C. (2003) Compliance with saliva sampling protocols: electronic monitoring reveals invalid cortisol daytime profiles in noncompliant subjects. Psychosomatic Medicine, 65, 313-319.

Loos, R., Derom, C., Vlietinck, R., et al (1998) The East Flanders Prospective Twin Survey (Belgium): a population-based register. Twin Research, I, 167-175.

Moffitt, T. E., Caspi, A. \& Rutter, M. (2005) Strategy for investigating interactions between measured genes and measured environments. Archives of General Psychiatry, 62, 473-481.
Myin-Germeys, I., van Os, J., Schwartz, J. E., et al (200I) Emotional reactivity to daily life stress in psychosis. Archives of General Psychiatry, 58, II37-II44.

Rutter, M. (2005) Environmentally mediated risks for psychopathology: research strategies and findings. Journal of the American Academy of Child and Adolescent Psychiatry, 44, 3-18.

Snijders, T. \& Bosker, R. (1999) Multilevel Analysis: An Introduction to Basic and Advanced Multilevel Modeling. Sage.

Van Os, J. \& Jones, P. B. (1999) Early risk factors and adult person-environment relationships in affective disorder. Psychological Medicine, 29, I055-1067. 